\title{
A NOTE ON THE SMALL-TIME DEVELOPMENT OF THE SOLUTION TO A COUPLED, NONLINEAR, SINGULAR REACTION-DIFFUSION SYSTEM
}

\author{
J. A. LEACH ${ }^{1}$
}

(Received 3 May, 2005; revised 10 January, 2006)

\begin{abstract}
In this paper, we consider a coupled, nonlinear, singular (in the sense that the reaction terms in the equations are not Lipschitz continuous) reaction-diffusion system, which arises from a model of fractional order chemical autocatalysis and decay, with positive initial data. In particular, we consider the cases when the initial data for the the dimensionless concentration of the autocatalyst, $\beta$, is of (a) $O\left(x^{-\lambda}\right)$ or (b) $O\left(e^{-\sigma x}\right)$ at large $x$ (dimensionless distance), where $\sigma>0$ and $\lambda$ are constants. While initially the dimensionless concentration of the reactant, $\alpha$, is identically unity, we establish, by developing the small-t (dimensionless time) asymptotic structure of the solution, that the support of $\beta(x, t)$ becomes finite in infinitesimal time in both cases (a) and (b) above. The asymptotic form for the location of the edge of the support of $\beta$ as $t \rightarrow 0$ is given in both cases.
\end{abstract}

2000 Mathematics subject classification: primary 35K57; secondary 41A60.

Keywords and phrases: reaction-diffusion, matched asymptotic expansions.

\section{Introduction}

In this paper we consider the following initial-boundary value problem for a coupled, nonlinear, singular (that is, non-Lipschitz) reaction-diffusion system, namely,

$$
\begin{array}{rlrl}
\alpha_{t} & =\alpha_{x x}-\alpha \beta^{m}, & \\
\beta_{t} & =\beta_{x x}+\alpha \beta^{m}-k \beta^{n}, & x, t>0, \\
\alpha(x, 0) & =1, \quad \beta(x, 0)=\beta_{0}(x), \quad x \geq 0, \\
\alpha_{x}(0, t) & =\beta_{x}(0, t)=0, & t>0,
\end{array}
$$

\footnotetext{
'Department of Mathematics, University of Reading, Whiteknights, Reading, Berkshire RG6 6AX, UK; e-mail: j.a.leach@reading.ac.uk.

(C) Australian Mathematical Society 2006, Serial-fee code 1446-1811/06
} 


$$
\begin{array}{lll}
\alpha(x, t) \stackrel{x \rightarrow \infty}{\longrightarrow} \hat{\alpha}_{\infty}(t), & t \geq 0 & \left(0 \leq \hat{\alpha}_{\infty}(t) \leq 1\right), \\
\beta(x, t) \stackrel{x \rightarrow \infty}{\longrightarrow} \hat{\beta}_{\infty}(t), & t \geq 0 & \left(0 \leq \hat{\beta}_{\infty}(t) \leq 1\right),
\end{array}
$$

where $0<n<m<1, k>0$ and $\beta_{0}(x)$ is a continuous, analytic, positive and monotone decreasing function in $x \geq 0$, with $\beta_{0}(x) \rightarrow 0$ as $x \rightarrow \infty$. In particular, we consider the following cases:

(a) Initial data for $\beta, \beta_{0}(x)$ that has algebraic decay rate as $x \rightarrow \infty$ and where

$$
\beta_{0}(x) \sim \begin{cases}\beta_{\infty} x^{-\lambda}+\operatorname{EST}(x) & \text { as } x \rightarrow \infty, \\ \tilde{\beta}_{0}+\sum_{l=1}^{\infty} \tilde{\beta}_{l} x^{l} & \text { as } x \rightarrow 0^{+},\end{cases}
$$

where $\lambda, \beta_{\infty}, \tilde{\beta}_{0}>0, \tilde{\beta}_{1}$ are constants and $\operatorname{EST}(x)$ denotes exponentially small terms in $x$ as $x \rightarrow \infty$.

(b) Initial data for $\beta, \beta_{0}(x)$ that has exponential decay rate as $x \rightarrow \infty$ and where

$$
\beta_{0}(x) \sim \begin{cases}\beta_{\infty} e^{-\sigma x}+O\left[e^{-f(x)}\right] & \text { as } x \rightarrow \infty, \\ \tilde{\beta}_{0}+\sum_{l=1}^{\infty} \tilde{\beta}_{l} x^{l} & \text { as } x \rightarrow 0^{+},\end{cases}
$$

for some $f(x)>O(x)$ as $x \rightarrow \infty$, where $\beta_{\infty}, \tilde{\beta}_{0}, \sigma>0$ and $\tilde{\beta}_{l}$ are constants.

Here $\alpha$ is the dimensionless concentration of the reactant, while $\beta$ is the dimensionless concentration of the autocatalyst.

A full description of the chemical model from which (1.1) arises, its analysis and a comprehensive review of the relevant literature may be found in [4] but is omitted here for brevity. Preliminary results concerning the system of singular reaction-diffusion equations can be found in [2], [5] and [6].

Our aim in this paper is to use the method of matched asymptotic expansions to develop the small-time asymptotic structure of the solution to initial-boundary value problem (1.1) with $0<n<m<1$ when the initial data, $\beta_{0}(x)$, of $\beta$ has algebraic or exponential decay rates as $x \rightarrow \infty$, given by (1.2) and (1.3) respectively. Throughout we use the nomenclature of the theory of matched asymptotic expansions, as given in Van Dyke [8] (see also Hinch [1], Lagerstrom [3] and Nayfeh [7] for an introduction to the theory of matched asymptotic expansions). We establish that in both cases the support of the solution for $\beta$ becomes finite in infinitesimal time. We conclude by presenting the asymptotic form for the location of the edge of the support of $\beta(x, t)$ as $t \rightarrow 0$ in both cases.

\section{Asymptotic solution as $t \rightarrow 0$}

In this section we develop the formal asymptotic structure to (1.1), for $n<m<1$, as $t \rightarrow 0$. The behaviour of the solution depends critically on the nature of $\beta_{0}(x)$ as 
$x \rightarrow \infty$. We will consider the cases when $\beta_{0}(x)$ has algebraic or exponential decay as $x \rightarrow \infty$ (given by (1.2) and (1.3) respectively) separately.

2.1. Initial data with algebraic decay as $x \rightarrow \infty$ We first consider region $I$, where $x=O(1)$ as $t \rightarrow 0$ and expand the solution to (1.1) as

$$
\begin{aligned}
& \alpha(x, t)=1+t \alpha_{1}(x)+O\left(t^{2}\right), \\
& \beta(x, t)=\beta_{0}(x)+t \beta_{1}(x)+O\left(t^{2}\right), \quad x, t>0,
\end{aligned}
$$

as $t \rightarrow 0$. On substitution into Equations (1.1a) and (1.1b) and applying initial conditions $(1.1 \mathrm{c})$ we readily obtain

$$
\begin{aligned}
& \alpha(x, t)=1-t \beta_{0}^{m}(x)+O\left(t^{2}\right) \\
& \beta(x, t)=\beta_{0}(x)+t\left(\beta_{0}^{\prime \prime}(x)+\beta_{0}^{m}(x)-k \beta_{0}^{n}(x)\right)+O\left(t^{2}\right)
\end{aligned}
$$

as $t \rightarrow 0$. Now, for $x \gg 1$, expansion (2.1b), with (1.2), takes the form

$$
\beta(x, t) \sim \beta_{\infty} x^{-\lambda}+t\left(\beta_{\infty} \lambda(\lambda+1) x^{-(\lambda+2)}-k \beta_{\infty}^{n} x^{-n \lambda}+\cdots\right)+\cdots
$$

as $t \rightarrow 0$, and we conclude that expansion (2.1b) becomes nonuniform when

$$
x=O\left(t^{-1 / \lambda(1-n)}\right)
$$

(that is, the dominant correction term in (2.1b) can be seen from (2.2) to become comparable with the leading order term in (2.1b) when $x=O\left(t^{-1 / \lambda(1-n)}\right)$ as $\left.t \rightarrow 0\right)$, when we observe, via (2.1), that

$$
\begin{aligned}
& \alpha=1-O\left(t^{(m-n+1) /(1-n)}\right), \\
& \beta=O\left(t^{1 /(1-n)}\right)
\end{aligned}
$$

as $t \rightarrow 0$. In order to continue the asymptotic structure we must therefore introduce a further region, which we refer to as region II. To examine region II, we introduce the scaled coordinate $\eta=x t^{1 / \lambda(1-n)}=O(1)$ and look, via (2.3), for asymptotic expansions of the form

$$
\begin{aligned}
& \alpha(\eta, t)=1-t^{(m-n+1) /(1-n)} \hat{\alpha}(\eta)+o\left(t^{(m-n+1) /(1-n)}\right), \\
& \beta(\eta, t)=t^{1 /(1-n)} \hat{\beta}(\eta)+o\left(t^{1 /(1-n)}\right)
\end{aligned}
$$

as $t \rightarrow 0$, with $\eta=O(1)$. On substitution of expansions (2.4) into Equations (1.1) (when written in terms of $\eta$ and $t$ ) we obtain the leading order problems for $\hat{\alpha}(\eta)$ and $\hat{\beta}(\eta)$ as

$$
\begin{gathered}
\eta \hat{\alpha}_{\eta}+\lambda(m-n+1) \hat{\alpha}=\lambda(1-n) \hat{\beta}^{m}, \quad 0<\eta<\infty, \\
\hat{\alpha}(\eta) \sim \beta_{\infty}^{m} \eta^{-m \lambda} \text { as } \eta \rightarrow 0
\end{gathered}
$$


and

$$
\begin{gathered}
\eta \hat{\beta}_{\eta}+\lambda \hat{\beta}+k \lambda(1-n) \hat{\beta}^{n}=0, \quad 0<\eta<\infty, \\
\hat{\beta}(\eta) \sim \beta_{\infty} \eta^{-\lambda} \text { as } \eta \rightarrow 0 .
\end{gathered}
$$

Conditions (2.5b) and (2.6b) arise from matching with region $I$ as $\eta \rightarrow 0$. The solution to $(2.6 \mathrm{a}),(2.6 \mathrm{~b})$ is readily obtained as

$$
\hat{\beta}(\eta)=\left[\beta_{\infty}^{(1-n)} \eta^{-\lambda(1-n)}-k(1-n)\right]^{1 /(1-n)}, \quad \eta>0 .
$$

An examination of (2.7) reveals that a weak singularity develops in $\hat{\beta}(\eta)$ as $\eta \rightarrow \eta_{c}^{-}$ (as $\eta$ approaches $\eta_{c}$ from below), where

$$
\eta_{c}=\left[\frac{\beta_{\infty}^{(1-n)}}{k(1-n)}\right]^{1 / \lambda(1-n)} .
$$

Now, via (2.7), the solution to (2.5) is given in terms of $\hat{\beta}$ by

$$
\hat{\alpha}(\eta)=\lambda(1-n) \eta^{-\lambda(m-n+1)} \int_{0}^{\eta} s^{\lambda(m-n+1)-1} \hat{\beta}^{m}(s) \mathrm{d} s, \quad 0<\eta<\eta_{c} .
$$

Further, we note that

$$
\begin{aligned}
& \hat{\alpha}(\eta)=\lambda(1-n) \mathscr{A} \eta^{-\lambda(m-n+1)}+O\left[\left(\eta_{c}-\eta\right)^{(m-n+1) /(1-n)}\right], \\
& \hat{\beta}(\eta)=\left[\frac{k(1-n)^{2} \lambda}{\eta_{c}}\right]^{1 /(1-n)}\left(\eta_{c}-\eta\right)^{1 /(1-n)}+O\left[\left(\eta_{c}-\eta\right)^{(2-n) /(1-n)}\right]
\end{aligned}
$$

as $\eta \rightarrow \eta_{c}^{-}$, where $\mathscr{A}=\int_{0}^{\eta_{c}} s^{\lambda(m-n+1)-1} \hat{\beta}^{m}(s) \mathrm{d} s$. Thus the support of $\beta(\eta, t)$ ends at $\eta=\eta_{c}$ in this region. However, in (2.4b) and (2.8b) the degree of $\left(\eta_{c}-\eta\right)$ as $\eta \rightarrow \eta_{c}^{-}$(which is $1 /(1-n)$ ), is too weak (we require a classical solution), and consideration of further terms in (2.4b) reveals a weak nonuniformity as $\eta \rightarrow \eta_{c}^{-}$. Therefore a further region is required to complete the asymptotic structure, in which $\eta=\eta_{c}+o(1)$ as $t \rightarrow 0$, and diffusion effects are retained at leading order to enable the appropriate behaviour to be achieved at the edge of the support. We label this region as region III and introduce the scaled coordinate $\bar{\eta}$ by

$$
\eta=\eta_{c}+t^{r} \bar{\eta}
$$

with $\gamma>0$ to be determined, and $\bar{\eta}=O(1)$ as $t \rightarrow 0$ in region III. An examination of (2.4) and (2.8) then determines that $\alpha=1-O\left(t^{(m-n+1) /(1-n)}\right)$ and $\beta=O\left(t^{(\gamma+1) /(1-n)}\right)$ in region III. Thus we expand as

$$
\begin{aligned}
& \alpha(\bar{\eta}, t)=1-t^{(m-n+1) /(1-n)} F(\bar{\eta})+o\left(t^{(m-n+1) /(1-n)}\right), \\
& \beta(\bar{\eta}, t)=t^{(\gamma+1) /(1-n)} H(\bar{\eta})+o\left(t^{(\gamma+1) /(1-n)}\right)
\end{aligned}
$$


as $t \rightarrow 0$ with $\bar{\eta}=O(1)$. On substituting (2.9b) into Equation (1.1b) (when written in terms of $\bar{\eta}$ and $t$ ), to retain diffusion terms at leading order requires

$$
\gamma=1+\frac{2}{\lambda(1-n)} \quad(>0),
$$

after which the leading-order problem is

$$
\begin{array}{ll}
H_{\bar{\eta} \bar{\eta}}-\frac{\eta_{c}}{\lambda(1-n)} H_{\dot{\eta}}-k H^{n}=0, & -\infty<\bar{\eta}<\bar{\eta}_{0}, \\
H\left(\bar{\eta}_{0}\right)=H_{\bar{\eta}}\left(\bar{\eta}_{0}\right)=0, & \\
H(\bar{\eta}) \sim\left[\frac{k(1-n)^{2} \lambda}{\eta_{c}}\right]^{1 /(1-n)}(-\bar{\eta})^{1 /(1-n)}, & \text { as } \bar{\eta} \rightarrow-\infty .
\end{array}
$$

Problem (2.10)-(2.12) is autonomous and can be studied in the $\left(H, H_{\bar{\eta}}\right)$ phase plane. It can readily be established (after minor modifications to [5, Appendix D]) that (2.10)(2.12) has a unique solution for each $\bar{\eta}_{0}$. Thus (2.10)-(2.12) does not fix a unique value of $\bar{\eta}_{0}$. In fact, $\bar{\eta}_{0}$ will be fixed by matching expansion (2.9b) (as $\bar{\eta} \rightarrow-\infty$ ) to expansion (2.4b) (as $\eta \rightarrow \eta_{c}^{-}$), when expansion (2.4b) is taken to next order. We observe that the solution to (2.10)-(2.12) is monotone decreasing in $-\infty<\bar{\eta}<\bar{\eta}_{0}$, and has

$$
H(\bar{\eta}) \sim\left[\frac{k(1-n)^{2}}{2(1+n)}\right]^{1 /(1-n)}\left(\bar{\eta}_{0}-\bar{\eta}\right)^{2 /(1-n)} \text { as } \bar{\eta} \rightarrow \bar{\eta}_{0}^{-},
$$

which has the required decay rate in $\left(\bar{\eta}_{0}-\bar{\eta}\right)$ as the edge of the support is approached. Consideration of further terms in this region shows that expansion (2.9b) remains uniform as $\bar{\eta} \rightarrow \bar{\eta}_{0}^{-}$.

We now return to the expansion for $\alpha$. On substituting (2.9) into Equation (1.1a) (when written in terms of $\bar{\eta}$ and $t$ ) we obtain at leading order the uncoupled boundary value problem for $F(\bar{\eta})$, given by

$$
\begin{aligned}
F_{\bar{\eta} \bar{\eta}}-\frac{\eta_{c}}{\lambda(1-n)} F_{\bar{\eta}}=0, & -\infty<\bar{\eta}<\infty, \\
F(\bar{\eta})>0, & -\infty<\bar{\eta}<\infty, \\
F(\bar{\eta}) \sim \lambda(1-n) \mathscr{A} \eta_{c}^{-\lambda(m-n+1)}, & \text { as } \bar{\eta} \rightarrow-\infty, \\
F(\bar{\eta}) \text { bounded, } & \text { as } \bar{\eta} \rightarrow \infty .
\end{aligned}
$$

Condition (2.13c) arises from matching with region II as $\bar{\eta} \rightarrow-\infty$. The solution to (2.13) is readily obtained as $F(\bar{\eta})=\lambda(1-n) \mathscr{A} \eta_{c}^{-\lambda(m-n+1)}$.

We note that the asymptotic structure of $\alpha(x, t)$ as $t \rightarrow 0$ does not end in this region. As $\bar{\eta} \rightarrow \infty$ we move out of the localised region III and re-emerge into region II 
$\left(\eta_{c}+o(1)<\eta<\infty\right)$ where $\alpha(\eta, t)=1-O\left(t^{(m-n+1) /(1-n)}\right)$ and $\beta(\eta, t) \equiv 0$. We note immediately, by consideration of $(2.5 \mathrm{a})$, that in this region (where $\eta_{c}+o(1)<\eta<\infty$ )

$$
\begin{aligned}
& \alpha(\eta, t)=1-\lambda(1-n) \mathscr{A} \eta^{-\lambda(m-n+1)} t^{(m-n+1) /(1-n)}+o\left(t^{(m-n+1) /(1-n)}\right), \\
& \beta(\eta, t) \equiv 0 .
\end{aligned}
$$

This completes the main asymptotic structure. In particular, we have that the edge of the support of $\beta(x, t), x=s(t)$, behaves as

$$
s(t) \sim \eta_{c} t^{-1 / \lambda(1-n)}+\bar{\eta}_{0} t^{1+1 / \lambda(1-n)}+\cdots
$$

as $t \rightarrow 0$. We observe from (2.14) that the edge of the support is contracting initially with speed

$$
\dot{s}(t) \sim-\frac{\eta_{c}}{\lambda(1-n)} t^{-1-1 / \lambda(1-n)}<0 \quad \text { as } \quad t \rightarrow 0 .
$$

Finally, we note that expansions (2.1) with (1.2) (as $x \rightarrow 0$ ) in region I do not, in general, satisfy the boundary conditions (1.1d) at $x=0$ and a further passive region is required in the neighbourhood of $x=0$ as $t \rightarrow 0$. The details of this region follow, after minor modifications, those given for region $\mathbf{I}_{0}$ in [6, Section 3].

A schematic representation of the location and thickness of the asymptotic regions as $t \rightarrow 0$ is given in Figure 1 .

2.2. Initial data with exponential decay as $x \rightarrow \infty \quad$ In this case expansion (2.1b) in region $I$ becomes nonuniform when $x=c(t)+O(1)$ where

$$
c(t)=-\frac{1}{\sigma(1-n)} \ln t
$$

as $t \rightarrow 0$, with $\alpha=1-O\left(t^{(m-n+1) /(1-n)}\right)$ and $\beta=O\left(t^{1 /(1-n)}\right)$ in region II. To examine region II, we introduce the coordinate $\eta=x-c(t)$ and look for expansions of the form

$$
\begin{aligned}
& \alpha(\eta, t)=1-t^{(m-n+1) /(1-n)} \bar{\alpha}(\eta)+o\left(t^{(m-n+1) /(1-n)}\right) \\
& \beta(\eta, t)=t^{1 /(1-n)} \bar{\beta}(\eta)+o\left(t^{1 /(1-n)}\right)
\end{aligned}
$$

as $t \rightarrow 0$, with $\eta=O(1)$. On substitution of expansions (2.15) into Equations (1.1a)-(1.1b) (when written in terms of $\eta$ and $t$ ) we obtain at leading order

$$
\begin{aligned}
\bar{\alpha}_{\eta}+\sigma(m-n+1) \bar{\alpha} & =\sigma(1-n) \bar{\beta}^{m}, \\
\bar{\beta}_{\eta}+\sigma \bar{\beta}+k \sigma(1-n) \bar{\beta}^{n} & =0,
\end{aligned}
$$




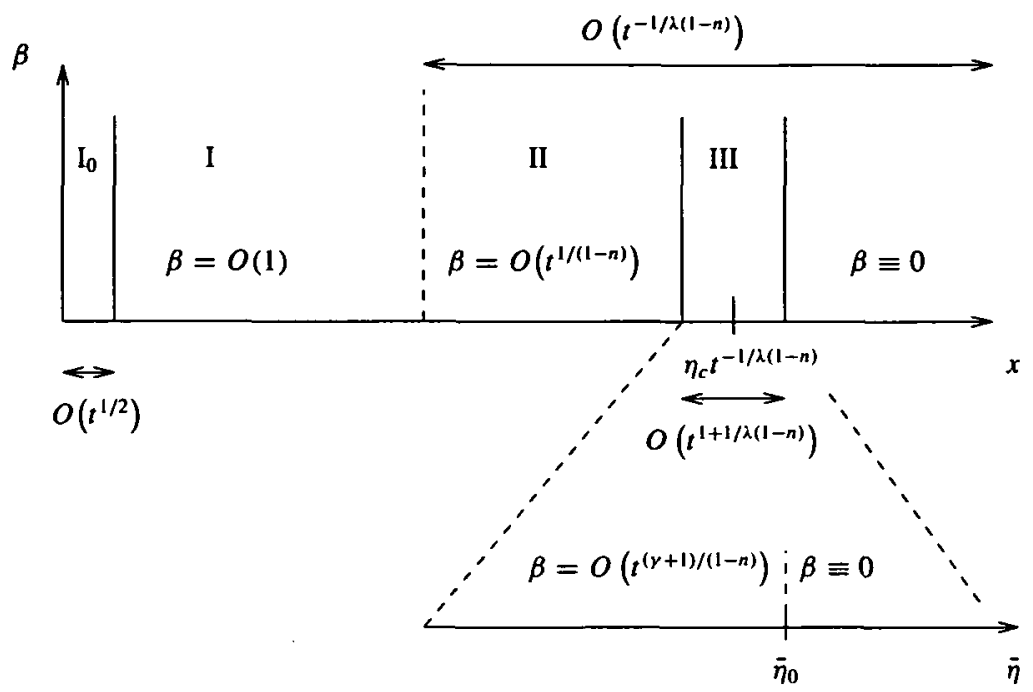

FIGURE 1. Schematic representation of the location and thickness of the asymptotic regions as $t \rightarrow 0$ in the case when the initial data has algebraic decay rate as $x \rightarrow \infty$. Note that in this case $\gamma=1+2 / \lambda(1-n)$ and $\alpha=1-O(t)$ in region I with $\alpha=1-O\left(t^{(m-n+1) /(1-n)}\right)$ in regions II and III.

where $\eta>-\infty$. Equations (2.16) are to be solved subject to matching with region I (as $\eta \rightarrow-\infty$ ), that is,

$$
\begin{array}{ll}
\bar{\alpha}(\eta) \sim \beta_{\infty}^{m} e^{-m \sigma \eta} & \text { as } \quad \eta \rightarrow-\infty \\
\bar{\beta}(\eta) \sim \beta_{\infty} e^{-\sigma \eta} & \text { as } \quad \eta \rightarrow-\infty .
\end{array}
$$

The solution to $(2.16 \mathrm{~b}),(2.17 \mathrm{~b})$ is readily obtained as

$$
\bar{\beta}(\eta)=\left[\beta_{\infty}^{(1-n)} e^{-\sigma(1-n) \eta}-k(1-n)\right]^{1 /(1-n)}, \quad \eta>-\infty .
$$

An examination of (2.18) reveals that a weak singularity develops in $\bar{\beta}(\eta)$ as $\eta \rightarrow \eta_{c}^{-}$, where

$$
\eta_{c}=\frac{1}{\sigma(1-n)} \ln \left[\frac{u_{\infty}^{(1-n)}}{k(1-n)}\right]
$$

Now, via (2.19), the solution to (2.16a), (2.17a), is given in terms of $\hat{\beta}$ by

$$
\bar{\alpha}(\eta)=\sigma(1-n) e^{-\sigma(m-n+1) \eta} \int_{-\infty}^{\eta} e^{\sigma(m-n+1) s} \hat{\beta}^{m}(s) \mathrm{d} s, \quad-\infty<\eta<\eta_{c} .
$$

Further, we note that

$$
\begin{aligned}
& \bar{\alpha}(\eta)=\sigma(1-n) \mathscr{B} e^{-\sigma(m-n+1) \eta}+O\left[\left(\eta_{c}-\eta\right)^{(m-n+1) /(1-n)}\right] \\
& \bar{\beta}(\eta)=\left[k \sigma(1-n)^{2}\right]^{1 /(1-n)}\left(\eta_{c}-\eta\right)^{1 /(1-n)}+o\left[\left(\eta_{c}-\eta\right)^{(2-n) /(1-n)}\right]
\end{aligned}
$$


as $\eta \rightarrow \eta_{c}^{-}$, where $\mathscr{B}=\int_{-\infty}^{\eta_{c}} e^{\sigma(m-n+1) s} \hat{\beta}^{m}(s) \mathrm{d} s$. Thus the support of $\beta(\eta, t)$ ends at $\eta=\eta_{c}$ in this region but as in Section 2.1 the degree of $\left(\eta_{c}-\eta\right)$ (in (2.15b)) as $\eta \rightarrow \eta_{c}^{-}$ is too weak, and consideration of further terms in $(2.15 \mathrm{~b})$ reveals a weak nonuniformity as $\eta \rightarrow \eta_{c}^{-}$[in particular, when $\eta=\eta_{c}+O(t)$ ]. We therefore introduce a final region, region III, to complete the asymptotic structure. The details of this region follow after minor modifications those given in Section 2.1 with now $\eta=\eta_{c}+\bar{\eta} t$ as $t \rightarrow 0$ with $\bar{n}=O(1)$. Consideration of (2.20b) determines that $\beta=O\left(t^{2 /(1-n)}\right)$ in region III (we will return to consideration of the $\alpha$ expansion later). Thus we expand as

$$
\beta(\bar{\eta}, t)=t^{2 /(1-n)} H(\bar{\eta})+o\left(t^{2 /(1-n)}\right)
$$

as $t \rightarrow 0$ with $\bar{\eta}=O(1)$. The leading-order problem is then given by

$$
\begin{array}{ll}
H_{\bar{\eta} \bar{\eta}}-\frac{1}{\sigma(1-n)} H_{\bar{\eta}}-k H^{n}=0, & -\infty<\bar{\eta}<\bar{\eta}_{0}, \\
H\left(\bar{\eta}_{0}\right)=H_{\bar{\eta}}\left(\bar{\eta}_{0}\right)=0, & \\
H(\bar{\eta}) \sim\left[k \sigma(1-n)^{2}\right]^{1 /(1-n)}(-\bar{\eta})^{1 /(1-n)}, \quad \text { as } \bar{\eta} \rightarrow-\infty .
\end{array}
$$

It can be readily established (after minor modifications to [5, Appendix D]) that (2.22)(2.24) has a unique solution for each $\bar{\eta}_{0}\left(\bar{\eta}_{0}\right.$ will be fixed by matching expansion (2.21) (as $\bar{\eta} \rightarrow-\infty)$ to expansion (2.15b) (as $\eta \rightarrow \eta_{c}^{-}$), when expansion (2.15b) is taken to next order). The solution to (2.22)-(2.24) is monotone decreasing in $-\infty<\bar{\eta}<\bar{\eta}_{0}$ and has

$$
H(\bar{\eta}) \sim\left[\frac{k(1-n)^{2}}{2(1+n)}\right]^{1 /(1-n)}\left(\bar{\eta}_{0}-\bar{\eta}\right)^{2 /(1-n)} \text { as } \bar{\eta} \rightarrow \bar{\eta}_{0}^{-},
$$

which has the required decay rate in $\left(\bar{\eta}_{0}-\bar{\eta}\right)$ as the edge of the support is approached. Consideration of further terms in this region shows that expansion (2.21) remains uniform as $\bar{\eta} \rightarrow \bar{\eta}_{0}^{-}$, and the asymptotic structure is complete.

We now return to the expansion for $\alpha$ in region III. Consideration of (2.20a) indicates that we should look for an expansion of the form

$$
\alpha(\bar{\eta}, t)=1-\sigma(1-n) \mathscr{B} e^{-\sigma(m-n+1) \eta_{c}} e^{-F(\bar{\eta}) t} t^{(m-n+1) /(1-n)}+o\left(t^{(m-n+1) /(1-n)}\right)
$$

as $t \rightarrow 0$. On substituting (2.25) into Equation (1.1a) (when written in terms of $\bar{\eta}$ and $t$ ) we obtain at leading order the uncoupled boundary value for $F(\bar{\eta})$, given by

$$
\begin{array}{ll}
F_{\bar{\eta} \dot{\eta}}-\frac{1}{\sigma(1-n)} F_{\bar{\eta}}=-\frac{m-n+1}{1-n}, & -\infty<\bar{\eta}<\infty, \\
F(\bar{\eta})>0, & -\infty<\bar{\eta}<\infty, \\
F(\bar{\eta}) \sim \sigma(m-n+1) \bar{\eta}, & \text { as } \bar{\eta} \rightarrow-\infty, \\
F(\bar{\eta}) \text { bounded, } & \text { as } \bar{\eta} \rightarrow \infty .
\end{array}
$$


Condition (2.26c) arises from matching with region II as $\bar{\eta} \rightarrow-\infty$. The solution to (2.26) is readily obtained as $F(\bar{\eta})=\sigma(m-n+1) \bar{\eta}$.

We note that the asymptotic structure of $\alpha(x, t)$ as $t \rightarrow 0$ does not end in this region. As $\bar{\eta} \rightarrow \infty$ we move out of region III and re-emerge into region II $\left(\eta_{c}+o(1)<\eta<\right.$ $\infty)$, where $\alpha(\eta, t)=1-O\left(t^{(m-n+1) /(1-n)}\right)$ and $\beta(\eta, t) \equiv 0$. We note immediately, by consideration of $(2.16 \mathrm{a})$, that in this region

$$
\begin{aligned}
& \alpha(\eta, t)=1-\sigma(1-n) \mathscr{B} e^{-\sigma(m-n+1) \eta} t^{(m-n+1) /(1-n)}+o\left(t^{(m-n+1) /(1-n)}\right), \\
& \beta(\eta, t) \equiv 0,
\end{aligned}
$$

where $\left(\eta_{c}+o(1)<\eta<\infty\right)$. This completes the main asymptotic structure. In particular, we have that the edge of the support of $\beta(x, t), x=s(t)$, behaves as

$$
s(t) \sim-\frac{1}{\sigma(1-n)} \ln t+\eta_{c}+O(t)
$$

as $t \rightarrow 0$ and we observe from (2.27) that the edge of the support is contracting initially with speed

$$
\dot{s}(t) \sim-\frac{1}{\sigma(1-n) t} \quad \text { as } \quad t \rightarrow 0 .
$$

Finally, we note that expansions (2.1) with (1.2) (as $x \rightarrow 0$ ) in region I do not, in general, satisfy the boundary conditions (1.1d) at $x=0$ and a further passive region is required in the neighbourhood of $x=0$ as $t \rightarrow 0$. The details of this region follow, after minor modifications, those given for region $\mathbf{I}_{0}$ in [6, Section 3].

A schematic representation of the location and thickness of the asymptotic regions as $t \rightarrow 0$ is given in Figure 2 .

\section{Summary}

In this paper we have demonstrated directly, via the method of matched asymptotic expansions, that the solution to the initial-boundary value problem (1.1) with $n<m<$ 1 together with initial data for $\beta$ that has infinite support with algebraic or exponential decay as $x \rightarrow \infty$ (given by conditions (1.2) and (1.3) respectively) develops finite support in $\beta$ in infinitesimal time. In particular, the edge of the support of $\beta, x=s(t)$, is given as follows:

(i) When the initial data of $\beta$ has algebraic decay rate as $x \rightarrow \infty$ by

$$
s(t) \sim \eta_{c} t^{-1 / \lambda(1-n)}+\bar{\eta}_{0} t^{1+1 / \lambda(1-n)}+\cdots \quad \text { as } t \rightarrow 0,
$$

where the constants $\eta_{c}$ and $\bar{\eta}_{0}$ are as described in Section 2.1. 


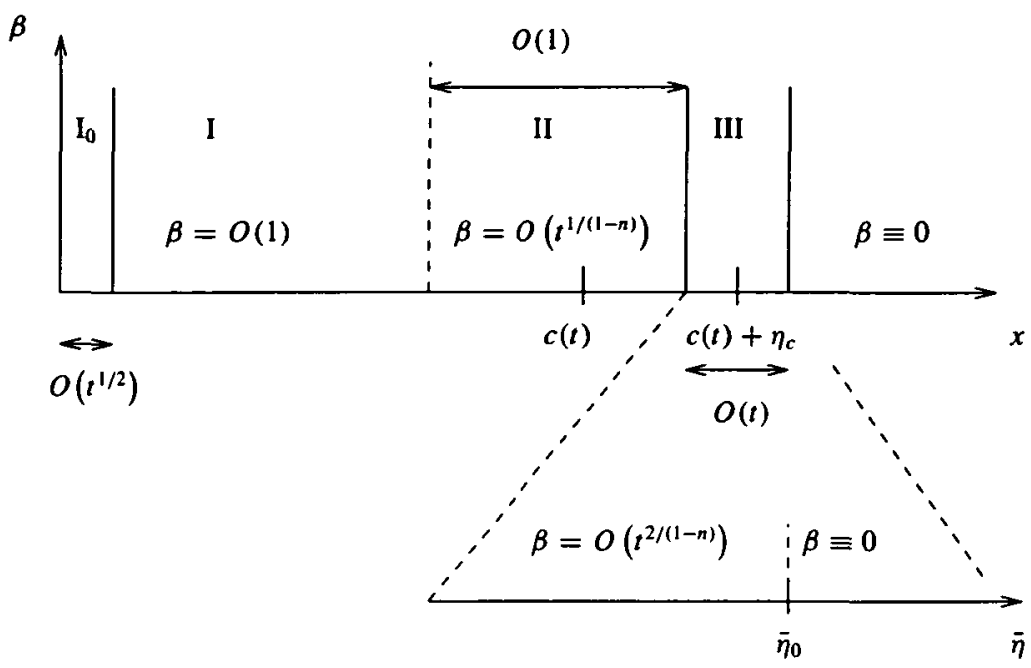

FIGURE 2. Schematic representation of the location and thickness of the asymptotic regions as $t \rightarrow 0$ in the case when the initial data has exponential decay rate as $x \rightarrow \infty$. We note that $\alpha=1-O(t)$ in region I and $\alpha=1-O\left(t^{(m-n+1) /(1-n)}\right)$ in regions II and III.

(ii) When the initial data of $\beta$ has exponential decay rate as $x \rightarrow \infty$ by

$$
s(t) \sim-\frac{1}{\sigma(1-n)} \ln t+\eta_{c}+O(t) \quad \text { as } t \rightarrow 0,
$$

where now $\eta_{c}$ is given in (2.19).

We note that in both cases the edge of the support is initially contracting.

Finally, we note that the asymptotic structure of the solution to (1.1) as $t \rightarrow 0$ when $m=n$ (with $k>1$ ) follows, after minor modifications, that given in Sections 2.1 and 2.2 when the initial data for $\beta$ has algebraic or exponential data as $x \rightarrow \infty$ respectively.

\section{References}

[1] E. J. Hinch, Perturbation Methods (Cambridge University Press, Cambridge, 1991).

[2] A. L. Kay, D. J. Needham and J. A. Leach, "Travelling waves for a coupled, singular reactiondiffusion system arising from a model of fractional order autocatalysis and decay", Nonlinearity 16 (2003) $735-770$.

[3] P. A. Lagerstrom and R. G. Casten, "Basic concepts underlying singular perturbation techniques", SIAM Review 14 (1972) 63-120.

[4] P. M. McCabe, J. A. Leach and D. J. Needham, "The evolution of travelling waves in fractional order autocatalysis with decay. I. Permanent form travelling waves", SIAM J. Appl. Math. 59 (1998) 870-899. 
[11] A note on the solution to a coupled, nonlinear, singular reaction-diffusion system

[5] P. M. McCabe, J. A. Leach and D. J. Needham, "The evolution of travelling waves in fractional order autocatalysis with decay. II. The initial boundary value problem", SIAM J. Appl. Math. 60 (2000) 1707-1748.

[6] P. M. McCabe, J. A. Leach and D. J. Needham, "On an initial-boundary-value problem for a coupled, singular reaction-diffusion system arising from a model of fractional order chemical autocatalysis and decay", Q. J. Mech. Appl. Math. 55 (2002) 511-560.

[7] A. H. Nayfeh, Introduction to Perturbation Techniques (Wiley-Interscience (John Wiley \& Sons), New York, 1981).

[8] M. Van Dyke, Perturbation Methods in Fluid Dynamics (Parabolic Press, Stanford, CA, 1975). 